\title{
Smear Positive Pulmonary Tuberculosis (TB) Patients Suspected to Have Drug Resistant TB in Programmatic Management of Drug Resistant TB Unit in Khyber Pakhtunkhwa, Pakistan
}

\author{
Arshad Javaid, ${ }_{1}$ Irfan Ullah, $, 2,{ }^{2}$ Mazhar Ali, ${ }^{1}$ Anila Basit,, Waqas Ahmad, ${ }^{4}$ Faisal Younis, ${ }^{3}$ Afsar Khan, ${ }^{1}$ \\ Ziaullah Shah, ${ }^{5}$ and Zahid Ahmad Butt ${ }^{6}$ \\ ${ }^{1}$ Programmatic Management of Drug resistant TB Pulmonology, Lady Reading Hospital Peshawar, Pakistan \\ ${ }^{2}$ Gomal Centre of Biochemistry and Biotechnology, Gomal University, Dera Ismail Khan, Khyber Pakhtunkhwa, Pakistan \\ ${ }^{3}$ Programmatic Management of Drug resistant TB Unit, TB Culture Laboratory, Mufti Mehmood Memorial Teaching Hospital, Dera Ismail Khan, Pakistan \\ ${ }^{4}$ Department of Mathematics, University of Science and Technology, Bannu \\ ${ }^{5}$ Department of Biotechnology and Microbiology, Sarhad University of Science and Information Technology Peshawar, Peshawar, Pakistan \\ ${ }^{6}$ School of Population and Public Health, University of British Columbia, Vancouver, British Columbia, Canada \\ "Corresponding author: Irfan Ullah, Gomal Centre of Biochemistry and Biotechnology, Gomal University, Dera Ismail Khan, Khyber Pakhtunkhwa, Pakistan. +92-3338333122, \\ Fax: +92-966747150, E-mail: irfan_btn@hotmail.com
}

Received 2016 October 04; Revised 2017 June 03; Accepted 2017 July 22.

\begin{abstract}
Background: Tuberculosis remains a major health problem with more than 3 million deaths and 9 million new cases annually. Pakistan ranks $5^{\text {th }}$ in the top 22 tuberculosis burden countries. Prevalence of all tuberculosis cases is 342 per 100,000 individuals in Pakistan.

Objectives: The objective of the present study was to assess the frequency and pattern of tuberculosis in a population from Khyber Pakhtunkhwa.

Methods: This prospective study was conducted in programmatic management of drug resistant tuberculosis unit lady reading hospital Peshawar, Pakistan between January, 2014 and December, 2014. A total of 1330 specimens from suspected drug resistant tuberculosis patients were analyzed by light-emitting diodes-fluorescence microscopy (LED-FM). The SPSS 18 software was used for data analysis.

Results: Of the 1330 drug resistant tuberculosis suspect patients tested by LED-FM microscopy, 824 (62\%) were smear positive for Mycobacterium and 306 (38\%) were negative. Mean age was 30.92 \pm 14.91 years. Out of smear positive cases, $462(56.1 \%)$ were female, 722 (87.6\%) were previously treated, and $446(54.1 \%)$ were in $<30$ years old. A statistically significant association was observed between female gender, previous tuberculosis treatment, and age category ( $<30$ years) with smear positive results.

Conclusions: Female gender, previous treatment, and young age ( $<30$ years) were significantly associated with smear positivity. Early detection and effective treatment of active tuberculosis cases is of paramount importance to reduce the burden of tuberculosis. Light-emitting diodes- fluorescence microscopy is the best method for early diagnosis of tuberculosis if performed by an experienced microbiologist, as it is reliable and inexpensive.
\end{abstract}

Keywords: Mycobacterium, Light-Emitting Diodes, Fluorescence Microscopy, Sputum

\section{Background}

Tuberculosis is a disease caused by Mycobacterium tuberculosis complex (MTBC) (1). Any organ of the body could be affected by MTBC, yet in two-thirds of cases it effects the lungs (Pulmonary tuberculosis) (1). Tuberculosis is commonly transmitted from person to person via droplet infection, mainly from sputum smear positive patients for acid fast bacilli (AFB) (2). Tuberculosis is a common public health problem in developing countries. It annually kills more people than any other infectious disease $(3,4)$. Currently, in less developed countries of Africa and Asia, the disease is highly prevalent where efforts to control tuberculosis are hampered by poor health systems, and in some countries by the high burden of co-infection with HIV (5).

The laboratory infrastructure is poor in these endemic countries. Sputum smear microscopy is a diagnostic tool for tuberculosis with low and variable sensitivity (6). Tuberculosis is one of the most important infectious diseases in the world and responsible for more than 3 million deaths and 9 million new cases, annually (4). Pakistan is at $5^{\text {th }}$ position in the top 22 tuberculosis burden countries. Prevalence of cases is 342 per 100,000 individuals (7).

The early detection and treatment of tuberculosis cases is very important for tuberculosis control because in Pakistan tuberculosis detection rate is only 63\% (8). Sputum smear microscopy (technique with low sensitivity and 
requiring a skilled practitioner) and chest radiography (lacking in specificity as well as sensitivity) are the diagnostic tests available in these settings (9). Pulmonary tuberculosis is commonly diagnosed by microscopy. Sputum induction is relatively easy to perform, generally welltolerated, and is frequently proposed as a technique to improve sample collection for tuberculosis diagnosis (2). Sensitivity is even lower where workload is high and technicians spend less time to examine the smears (10).

In 2006, a systematic review was published, in which specificities of fluorescence microscopy and conventional Ziehl-Neelsen microscopy was compared. This review reported that smear by fluorescence microscopy could improve sensitivity of smear microscopy by $10 \%$ over the conventional Ziehl-Neelsen microscopy (11).

\section{Objectives}

This study aimed at investigating the frequency and pattern of tuberculosis in Khyber Pakhtunkhwa, Pakistan using light-emitting diodes-fluorescence microscopy(LEDFM).

\section{Methods}

\subsection{Ethics Statement}

This study was approved by research and ethics committee of the post graduate medical institute (PGMI) (No: 354/ PGMI/ LRH/2013/245), lady reading hospital (LRH), Peshawar, Pakistan.

\subsection{Clinical Specimens}

The present study was conducted at programmatic management of drug resistant tuberculosis (PMDT) Unit LRH Peshawar, Pakistan. A total of 1330 specimens were collected from suspected patients of drug resistant tuberculosis at PMDT, LRH, from January 2014 to December 2014, and included in this study. The referrals of these specimens were according to the guidelines of the national tuberculosis program for referral of specimens for presumptive drug resistant tuberculosis. Direct sputum lightemitting diodes- fluorescence microscopy (LED-FM) was performed routinely in the clinic's laboratory. Patients submitted their sputum specimens after providing a written informed consent. Instructions were provided for the patients for the production of good quality sputum specimens (12).

\subsection{Preparation of Slides}

Smears were stained for LED-FM with $0.1 \%$ auraminerhodamine acid fast staining for a minimum of 15 minutes, which were then de-stained with $1 \% \mathrm{HCl}$ in alcohol for 2 minutes and then counterstained with potassium permanganate for 60 seconds and examined under LED-FM (ZEISS, Primo star, primo plan-achromat) (magnification, X400). The number of acid fast bacilli (AFB) examined per standard length (i.e. $1 \mathrm{~cm}$ large and $2 \mathrm{~cm}$ long) was reported. A length corresponded under 1000 and 400 magnification was 100 and 20 fields magnification respectively (13). As LED-FM slides should be examined within 24 hours, as part of the internal quality control (IQC), all LED-FM slides were controlled by the laboratory supervisor during one day (chosen randomly) per week. The second reader on the same day blindly examined a random sample of 200 auramine stained smears, to measure the inter-reader reliability of the LED-FM.

The sputum smear results were graded as per world health organization (WHO) protocol as follows (14):

1. Negative results were recorded, if no AFB was found in one length

2. If 1-2 AFB in one length was found, confirmation required by another technician or another smear was prepared, stained, and read.

3. Actual count or exact figure was recorded, if 3-24 AFB was found in one length.

4. (+) was recorded, if 1 to $6 \mathrm{AFB}$ was found in one field.

5. $(++)$ was recorded, if 7 to $60 \mathrm{AFB}$ were found per field.

6 . $(+++)$ was recorded, if more than 60 AFB was found per field.

\subsection{Statistical Analysis}

The data were analyzed using statistical package for social sciences (SPSS 18) for windows (SPSS Inc., Chicago, IL, USA). Data are presented as frequencies for categorical variables, and by mean + standard deviation for numerical variables. Association between categorical variables was observed using the Chi-square test (and Fisher exact test wherever necessary). The criterion for significance was set at $\mathrm{P}<0.05$ based on a two-sided test.

\section{Results}

A total of 1330 tuberculosis suspects were included in the final results. Overall, the proportion of females was relatively higher $(n=728,54.7 \%)$ than males (Table 1$)$. The mean age of patients was $39.7(S D=18.5)$ years, ranging from 10 to 80 years. The most suspected patients were those aged less than 30 of years ( $n=642,48.3 \%$ ), followed 
by those aged between 31 and 60 years ( $n=520,39.1 \%$ ) (Table 1). A total of 1138 patients (85.6\%) also reported a previous history of tuberculosis, which was treated with drugs while 192 (14.4\%) were newly diagnosed patients (Table 1). Of the 1330 suspected patients tested by LED microscopy, 824 (62\%) were positive for Mycobacterium and 506 (38\%) were negative. Regarding positive cases, 48 (3.6\%) were scanty, 304 (22.9) 1+, 276 (20.8\%) 2+, and 196 (14.7\%) 3+ (Table 2).

Table 1. Demographics of Enrolled Suspects

\begin{tabular}{|cc|}
\hline \multicolumn{1}{l}{ Variables } & Values $^{\mathbf{a}}$ \\
\hline Gender & \\
\hline Male & $602(45.3)$ \\
\hline Female & $728(54.7)$ \\
\hline Age, $\mathbf{~}$ & $39.7 \pm 18.5$ \\
\hline$<30$ & $642(48.3)$ \\
\hline 31 - 60 & $520(39.1)$ \\
\hline$>65$ & $168(12.6)$ \\
\hline Tuberculosis history & $192(14.4)$ \\
\hline New patients & $1138(85.4)$ \\
\hline
\end{tabular}

${ }^{\mathrm{a}}$ Values are expressed as No. (\%) or means $\pm \mathrm{SD}$.

Table 2. Sputum Smear Results of Tuberculosis Suspects

\begin{tabular}{lc}
\hline Smear Grading & Frequency (\% Age) \\
\hline Negative & $506(38)$ \\
Scanty & $48(3.6)$ \\
\hline $\mathbf{1}+$ & $304(22.9)$ \\
\hline $\mathbf{2}+$ & $276(20.8)$ \\
\hline $3+$ & $196(14.7)$ \\
\hline Total & $1330(100)$ \\
\hline
\end{tabular}

Univariate analysis revealed that the prevalence of tuberculosis was relatively higher in females $(n=462,54.7 \%)$ than males (Table 3 ). The previously treated patients $(\mathrm{n}=$ $722,85.6 \%$ ) were more likely to experience tuberculosis as compared to newly diagnosed patients (Table 3 ). Most of the tuberculosis cases $(n=446,48.3 \%$ ) were found among patients aged less than 30 years as compared to other age groups (Figure 1 and Table 3 ). Individuals, who were $<30$ years old $(\mathrm{P}<0.001)$ had a statistically significant association with tuberculosis as compared to the age group of more than 60 years.

\section{Discussion}

The early detection and effective treatment of active tuberculosis cases in highly prevalent countries is important for tuberculosis control, especially in pulmonary tuberculosis cases. The main reason for the high risk of infection, death rate, and drug resistance cases in Pakistan may be due to delay in diagnosis and failure to cure high proportion of pulmonary smear positive cases. Pakistan is at the $5^{\text {th }}$ position in the 22 top tuberculosis burden countries (15), where $26 \%$ of the patients have no knowledge about tuberculosis disease before diagnosis and 10\% of the general population is not aware of the disease (16). Previous studies show that $56 \%$ to $99 \%$ of people were aware of tuberculosis disease in India (17-19). In the present study, 62\% were smear positive among suspected drug resistant tuberculosis patients, which is worrying as poor knowledge may be the reason for high burden of tuberculosis in Pakistan.

In Pakistan, diagnosis of tuberculosis depends on radiographic changes, microscopy, and culture (where available). Ziehl-Neelsen staining is a simple, rapid, and inexpensive test for diagnosis of pulmonary tuberculosis yet has low sensitivity. In 2010, the WHO recommended that LED-FM should replace conventional FM in all settings, and that LED microscopy should be phased as an option for conventional Ziehl-Neelsen microscopy (20).

Out of the total suspected cases in the present study, $62 \%$ were smear positive in the present study; however, they were not confirmed by culture for Mycobacterium tuberculosis. The current results are similar to that of the previous study by Zaman et al. who found 52\% smear positivity (21). Females were $63.5 \%$ in smear positive cases in this study. The present study was in line with other previous studies, in which females were more affected by tuberculosis than males (22-27). Zaman et al. and Akhtar et al. also noted that tuberculosis was more prevalent in females than in males $(21,28)$. Our results are also in agreement with the results of Ahmad et al. who reported tuberculosis to be more frequent in females as compared to males (29). Long et al. reported that females waited almost twice as compared to males to visit health care centers or hospitals in Vietnam for diagnosis (30). Females are busy with the care of children and working at home, thereby neglect their illness and do not refer to the health system when they are severely affected by the disease (31). In some societies, the occurrence of tuberculosis infection in unmarried females makes it difficult to find life partners and in married females, they are forced to divorce (32).

Age is also an important factor in the prevalence of tuberculosis. Association between tuberculosis and age is not consistent among studies, as researchers used differ- 


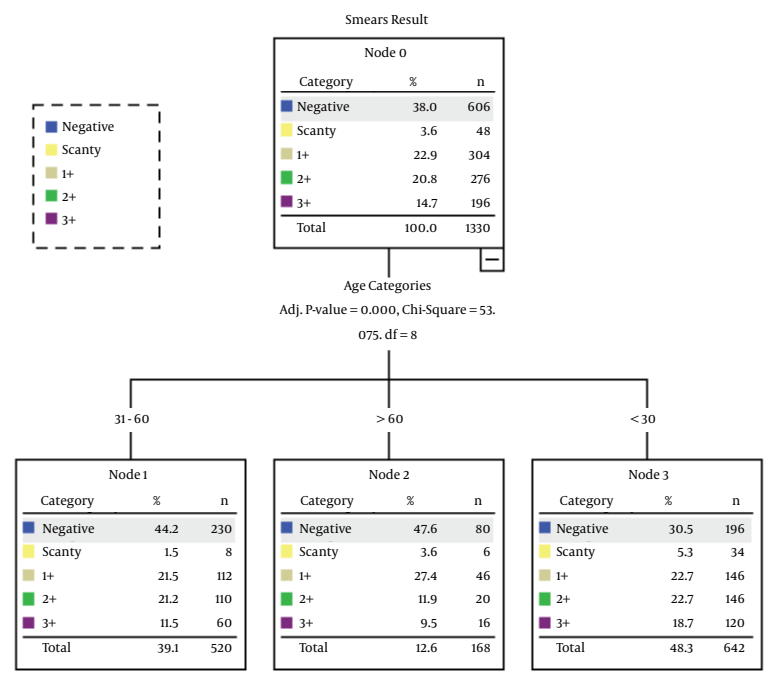

Figure 1. The Association Between Patients' Age and Smear Results

Table 3. The Association Between Patients' Demographics and Smear Results

\begin{tabular}{|c|c|c|c|c|c|c|c|c|}
\hline & & \multicolumn{5}{|c|}{ Smear Results } & \multirow[t]{2}{*}{ Total (\%Age) } & \multirow[t]{2}{*}{ PValue } \\
\hline & & Negative (\%Age) & Scanty (\%Age) & 1+ (\%Age) & 2+ (\%Age) & $3+(\%$ Age $)$ & & \\
\hline \multirow{2}{*}{ Genders } & Female & $266(36.5)$ & $26(3.6)$ & $162(22.3)$ & $144(19.8)$ & $130(17.9)$ & $728(100)$ & \multirow{2}{*}{0.014} \\
\hline & Male & $240(39.9)$ & $22(3.7)$ & $142(23.6)$ & $132(21.9)$ & $66(11)$ & $602(100)$ & \\
\hline \multirow{2}{*}{$\begin{array}{l}\text { Tuberculosis } \\
\text { History }\end{array}$} & New Patient & $90(46.9)$ & $8(4.2)$ & $30(15.6)$ & $42(21.9)$ & $22(11.5)$ & $192(100)$ & \multirow{2}{*}{0.020} \\
\hline & $\begin{array}{l}\text { Previously treated } \\
\text { patients }\end{array}$ & $416(36.6)$ & $40(3.5)$ & $274(24.1)$ & $234(20.6)$ & $174(15.3)$ & $1138(100)$ & \\
\hline \multirow{3}{*}{ Age Categories, y } & $<30$ & $196(30.5)$ & $34(5.3)$ & $146(22.7)$ & $146(22.7)$ & $120(18.7)$ & $642(100)$ & \multirow{3}{*}{$<0.001$} \\
\hline & $31-60$ & $230(44.2)$ & $8(1.5)$ & $112(21.5)$ & $110(21.2)$ & $60(11.5)$ & $520(100)$ & \\
\hline & $>60$ & $80(47.6)$ & $6(3.6)$ & 4627.4 & $20(11.9)$ & $16(9.5)$ & $168(100)$ & \\
\hline
\end{tabular}

ent age group cut-offs from one another. However, it has been reported that tuberculosis patients were likely to be younger. Age category ( $<30$ years) had a statistically significant association with smear positive results. Smear positive cases (48.3\%) were present in individuals aged $<30$ years while $12.6 \%$ were present in patients aged more than 60 years old. In Pakistan, most tuberculosis cases occurred in the most economically productive age group $(33,34)$. The authors hypothesize that this difference could be due to the fact that younger aged patients are often busy in routine activities, such as study or work as compared to elderly whose lifestyles are sedentary in our setting so that they are unable to maintain compliance. Another reason could be that they had greater exposure to tuberculosis patients as compared to elderly. However, this finding requires further investigations.
Previous tuberculosis treatment has statistically significant positive association with smear positive results. In the current study, the affected patients of tuberculosis were more commonly found amongst previously treated patients as compared to newly diagnosed patients. This finding is corroborated by a study by Denboon et al. (35) and may be due to low treatment success rate. Delays in diagnosis and accessing care are factors that may contribute to tuberculosis transmission. The current results indicate that there may be a considerable number of incompletely cured patients or previously treated tuberculosis cases that contribute to transmission of tuberculosis. In Pakistan, infants are vaccinated with Bacille Calmette-Guerin (BCG) to protect from tuberculosis disease in children (36). Danish BCG strain 1331 (Copenhagen, State Serum Institute) was used up until year 2007 in Pakistan (37). However, in- 
spite of vaccination, tuberculosis remains a considerable public health issue in Pakistan.

The current study demonstrated the utility of LED-FM in resource poor settings, like Pakistan. Light-emitting diodes-fluorescence microscopy could be used in places where high technology tests are not available in Pakistan. Currently, 1401 Microscopy centers are working and in these centers, Ziehl-Neelsen microscopy is mostly used, which is less sensitive than LED-FM. Light-emitting diodesfluorescence microscopy requires minimal consumption of power supply, correct installation, and maintenance, and has extraordinary long-life span and does not produce Ultra Violet light. This makes it more suitable for use in high burden and resource-limited countries (38). Smear reading with LED-FM (mean time $=1$ minute) is 3 times faster as compared to Ziehl-Neelsen (mean time = 3 minute) (39). According to a previous study, this study saved $25 \%$ to $65 \%$ of time, when using LED-FM (40). Therefore, the introduction of FM microscopy would allow better microscopy and significantly reduce laboratory workload with the same human resources as compared to ZiehlNeelsen microscopy. Previous research has suggested that strong affinity of carbol auramine to mycolic acid compared to carbol fuchsin (41) would increase sensitivity of LED-FM for AFB.

Both xylene and immersion oil used for cleaning off with Ziehl-Neelsen are expensive and through misuse or poor quality often cause damage to the objective of the Ziehl-Neelsen microscopy (42). The initial investment for LED-FM is considerable, yet the running costs are lower than Ziehl-Neelsen as there is no need of immersion oil or xylene, replacement of bulbs or objective (43). Lightemitting diodes-fluorescence microscopy offers many advantages with appropriate equipment. The solution is easier to prepare and the staining technique is easier to control (11), as the quality of commercial auramine powder is less variable than that of basic fuchsine (44). Furthermore, if electrical and mechanical parts are high quality than it is trouble free for a long time, seeing 20,000 hours life span of LED-FM light source (42). This study confirms acceptability of LED-FM and the advantage of using this technique in peripheral laboratories. For the National Tuberculosis Control Program, these are all significant thoughts in scalingup LED-FM with limited resources.

\subsection{Conclusions}

In the present study, female gender, previous treatment and younger age category were significantly associated with smear positivity. The early detection and effective treatment of active tuberculosis cases under supervision is important for tuberculosis control. The LED-FM microscopy is the best method for early diagnosis of tubercu- losis if performed by an experienced microbiologist, as it is reliable and inexpensive.

\section{Footnotes}

Authors' Contribution: Arshad Javaid and Irfan Ullah contributed equally as 1st author on this paper. Conceived and designed the experiments: Irfan Ullah, Arshad Javaid; performed the experiments: Irfan Ullah; analyzed the data: Irfan Ullah, Mazhar Ali, Anila Basit, Waqas Ahmad, Faisal Younis, Afsar Khan, Ziaullah Shah; wrote the paper: Irfan Ullah, Arshad Javaid, critically Reviewed/Revised the paper: Zahid Ahmad Butt.

Funding/Support: This research did not receive any specific grant from funding agencies in the public, commercial or non-profit sectors.

Competing Interest: The authors declare no competing interests.

\section{References}

1. Forrellad MA, Klepp LI GA, Sabio GJ, Morbidoni HR, Santangelo MP. Virulence factors of the Mycobacterium tuberculosis complex. Virulence 2013. Available from: http://www.tandfonline.com/doi/abs/10. 4161/viru.22329.

2. Sia IG, Wieland ML, editors. Current concepts in the management of tuberculosis. Mayo Clinic Proceedings. 2011; Elsevier; pp. 348-61.

3. Khan M, Mehreen S, Basit A, Khan R, Jan F, Ullah I, et al. Characteristics and treatment outcomes of patients with multi-drug resistant tuberculosis at a tertiary care hospital in Peshawar, Pakistan. Saudi Med J. 2015;36(12):1463-71. doi: 10.15537/smj.2015.12.12155.

4. World Health Organization . Global Tuberc Rep 2015. Geneva: World Health Organization; 2015.

5. Mohajan HK. Tuberculosis is a fatal disease among some developing countries of the world. Am J Infect Dis Microbiol. 2015;3(1):18-31.

6. Parsons LM, Somoskovi A, Gutierrez C, Lee E, Paramasivan CN, Abimiku A, et al. Laboratory Diagnosis of Tuberculosis in ResourcePoor Countries: Challenges and Opportunities. Clin Microbiol Rev. 2011;24(2):314-50. doi: 10.1128/cmr.00059-10.

7. Ullah I, Javaid A, Tahir Z, Ullah O, Shah AA, Hasan F, et al. Pattern of Drug Resistance and Risk Factors Associated with Development of Drug Resistant Mycobacterium tuberculosis in Pakistan. PLoS One. 2016;11(1):e0147529. doi: 10.1371/journal.pone.0147529. [PubMed: 26809127].

8. Ullah I, Javaid A, Masud H, Ali M, Basit A, Ahmad W, et al. Rapid detection of Mycobacterium tuberculosis and rifampicin resistance in extrapulmonary tuberculosis and sputum smear-negative pulmonary suspects using Xpert MTB/RIF. J Med Microbiol. 2017;66(4):412-8. doi: 10.1099/jmm.0.000449. [PubMed: 28425873].

9. Perkins MD, Cunningham J. Facing the crisis: improving the diagnosis of tuberculosis in the HIV era. J Infect Dis. 2007;196 Suppl 1:S15-27. doi: 10.1086/518656. [PubMed: 17624822].

10. Yassin MA, Cuevas LE. How many sputum smears are necessary for case finding in pulmonary tuberculosis? Trop Med Int Health. 2003;8(10):927-32. [PubMed: 14516304].

11. Mase SR, Ramsay A, Ng V, Henry M, Hopewell PC, Cunningham J, et al. Yield of serial sputum specimen examinations in the diagnosis of pulmonary tuberculosis: a systematic review. Int J Tuberc Lung Dis. 2007;11(5):485-95. [PubMed: 17439669]. 
12. Alisjahbana B, van Crevel R, Danusantoso H, Gartinah T, Soemantri ES, Nelwan RH, et al. Better patient instruction for sputum sampling can improve microscopic tuberculosis diagnosis. Int J Tuberc Lung Dis. 2005;9(7):814-7. [PubMed: 16013780].

13. Mabaera B, Lauritsen JM, Katamba A, Laticevschi D, Naranbat N, Rieder HL. Making pragmatic sense of data in the tuberculosis laboratory register. Int JTuberc Lung Dis. 2008;12(3):294-300. [PubMed:18284835].

14. Wallenburg HC. [Therapeutic aspects of chronic placental insufficiency]. Arch Gynakol. 1975;219(1-4):369. [PubMed: 2136].

15. Ullah I, Shah AA, Basit A, Ali M, Ullah U, Ihtesham M, et al. Rifampicin resistance mutations in the 81 bp RRDR of rpo $B$ gene in Mycobacterium tuberculosis clinical isolates using Xpert MTB/RIF in Khyber Pakhtunkhwa, Pakistan: a retrospective study. BMC Infect Dis. 2016;16(1):413. doi: 10.1186/s12879-016-1745-2.

16. Khan JA, Irfan M, Zaki A, Beg M, Hussain SF, Rizvi N. Knowledge, attitude and misconceptions regarding tuberculosis in Pakistani patients. J Pak Med Assoc. 2006;56(5):211-4. [PubMed: 16767946].

17. Kar M, Logaraj M. Awareness, attitude and treatmnt seeking behaviour regarding tuberculosis in a rural area of Tamil Nadu. Indian J Tuberc. 2010;2:3-6.

18. Zander J, Nitsch B. [Diagnosis and treatment of early pregnancy complications (proceedings)].Arch Gynakol. 1977;224(1-4):59-79. [PubMed: 23741].

19. Cullen MJ. The jumping mechanism of Xenopsylla cheopis. II. The fine structure of the jumping muscle. Philos Trans $R$ Soc Lond B Biol Sci. 1975;271(914):491-7. [PubMed: 1805].

20. Organization WH . Fluorescent light-emitting diode ( LED ) microscopy for diagnosis of tuberculosis Policy statement. ; 2011.

21. Khattak MI, Muhammad A, Khan N, Zaman M. Frequency of sputum positive AFB cases among patients of pulmonary tuberculosis in tertiary care hospitals of northern Pakistan. J Ayub Med Coll Abbottabad. 2010;22(2):56-60. [PubMed: 21702267].

22. Codlin AJ, Khowaja S, Chen Z, Rahbar MH, Qadeer E, Ara I, et al. Short report: Gender differences in tuberculosis notification in Pakistan. Am J Trop Med Hyg. 2011;85(3):514-7. doi: 10.4269/ajtmh.2011.10-0701. [PubMed: 21896814].

23. Dogar OF, Shah SK, Chughtai AA, Qadeer E. Gender disparity in tuberculosis cases in eastern and western provinces of Pakistan. BMC Infect Dis. 2012;12:244. doi: 10.1186/1471-2334-12-244. [PubMed: 23040242].

24. Noori MY, Ali Z, Mughal MN, Sharafat S, Masroor M, Khanani R. Gender distribution of smear positive tuberculosis cases at a tertiary care chest diseases centre in karachi. Journal of the Dow University of Health Sciences Karach. 2015;9(1):17-19.

25. Ahmad T, Zohaib MD, Zaman Q, Saifullah MAJ, Aryal S, Pandey S, et al. Prevalence of tuberculosis infection in general population of district Dir (lower) Pakistan. Middle East J Sci Res. 2015;23(1):14-7.

26. Shafee M, Abbas F, Ashraf M, Mengal MA, Kakar N, Ahmad Z, et al. Hematological profile and risk factors associated with pulmonary tuberculosis patients in Quetta, Pakistan. PakJMed Sci. 2014;30(1):36-40. doi: $10.12669 /$ pjms.301.4129.

27. Shams S, Rahman A. Prevalence of Tuberculosis in District Khar Bajaur Agency, Khyber Pakhtunkhwa Pakistan. Biochem Analyt Biochem. 2014;3(2) doi: 10.4172/2161-1009.1000148.

28. Akhtar T, Safi MI, Ahmed N. Management of tuberculosis by practitioners of Peshawar. J Pak Med Assoc. 1994;44(12):280-2. [PubMed: 7861583].
29. Ahmad T, Jadoon MA, Khattak MNK. Prevalence of sputum smear positive pulmonary tuberculosis at Dargai, District Malakand, Pakistan: A four year retrospective study. Egypt J lChest Dis Tuberculosis 2016;65(2):461-4. doi:10.1016/j.ejcdt.2015.12.004.

30. Long NH, Johansson E, Diwan VK, Winkvist A. Fear and social isolation as consequences of tuberculosis in VietNam: a gender analysis. Health Policy. 2001;58(1):69-81. [PubMed: 11518602].

31. Atre SR, Kudale AM, Morankar SN, Rangan SG, Weiss MG. Cultural concepts of tuberculosis and gender among the general population without tuberculosis in rural Maharashtra, India. Trop Med Int Health. 2004;9(11):1228-38. doi: 10.1111/j.1365-3156.2004.01321.x. [PubMed: 15548321].

32. Waisbord Washington DC 2004. S. Behavioral Barriers in Tuberculosis Control: A Literature Review. Washington, DC, USA: ,The CHANGE Project/Academy for Educational Development.; 2004

33. WHO . Global Tuberculosis Report 2013 World Heal Organ; 2013 Available from: http://apps.who.int/iris/bitstream/10665/91355/1/ 9789241564656_eng.pdf.

34. Ullah I, Shah AA, Tahir Z, Hasan F, Ayub N. Detection of Mycobacterium tuberculosis from Clinical Specimens by Conventional and Molecular Technique in Punjab , Pakistan. Glob Vet. 2014;13(6):1002-9.

35. den Boon S, van Lill SW, Borgdorff MW, Enarson DA, Verver S, Bateman ED, et al. High prevalence of tuberculosis in previously treated patients, Cape Town, South Africa. Emerg Infect Dis. 2007;13(8):1189-94. doi: 10.3201/eid1308.051327. [PubMed: 17953090].

36. Hasan Z, Irfan M, Khan JA, Jahangir SK, Haris M, Ashraf M, et al. BCG vaccination is associated with decreased severity of tuberculosis in Pakistan. Int $J$ Mycobacteriol. 2012;1(4):201-6. doi 10.1016/j.ijmyco.2012.10.007. [PubMed: 26785624].

37. Zwerling A, Behr MA, Verma A, Brewer TF, Menzies D, Pai M. The BCG World Atlas: a database of global BCG vaccination policies and practices. PLoS Med. 2011;8(3):e1001012. doi: 10.1371/journal.pmed.1001012. [PubMed: 21445325].

38. Stop TB Partnership and World Health Organization . New laboratory diagnostic tools for tuberculosis control. Retooling task force. Geneva: WHO; 2008.

39. Bonnet M, Ramsay A, Githui W, Gagnidze L, Varaine F, Guerin PJ Bleach sedimentation: an opportunity to optimize smear microscopy for tuberculosis diagnosis in settings of high prevalence of HIV. Clin InfectDis. 2008;46(11):1710-6. doi:10.1086/587891.[PubMed:18444789].

40. Marais BJ, Brittle W, Painczyk K, Hesseling AC, Beyers N, Wasserman $\mathrm{E}$, et al. Use of light-emitting diode fluorescence microscopy to detect acid-fast bacilli in sputum. Clin Infect Dis. 2008;47(2):203-7. doi 10.1086/589248. [PubMed: 18532893].

41. Ba F, Rieder HL. A comparison of fluorescence microscopy with the Ziehl-Neelsen technique in the examination of sputum for acid-fast bacilli. Int J Tuberc Lung Dis. 1999;3(12):1101-5. [PubMed: 10599014].

42. Lumb R, Van Deun A, Kelly P, Bastian I. Not all microscopes are equal. Int J Tuberc Lung Dis. 2006;10(2):227-9. [PubMed: 16499266].

43. Angra P, Becx-Bleumink M, Gilpin C, Joloba M, Jost K, Kam KM, et al Ziehl-Neelsen staining: strong red on weak blue, or weak red under strong blue? Int J Tuberc Lung Dis. 2007;11(11):1160-1. [PubMed: 17958975].

44. Van Deun A, Hossain MA, Gumusboga M, Rieder HL. Ziehl-Neelsen staining: theory and practice. Int J Tuberc Lung Dis. 2008;12(1):108-10. [PubMed: 18173887]. 